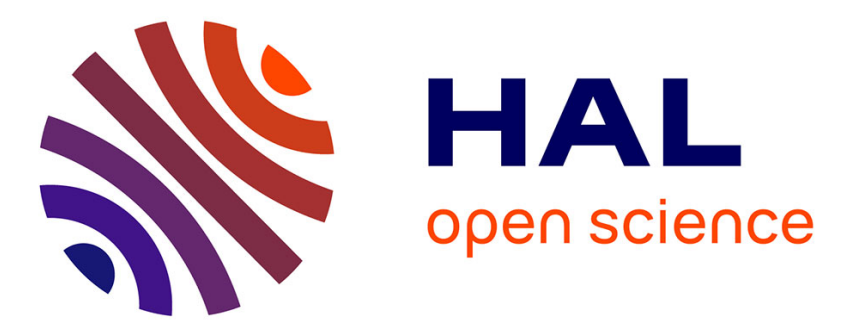

\title{
Il possessivo e gli avverbi di luogo nel fumetto italiano: dal progetto di ricerca all'uso pedagogico
}

\author{
Virginie Culoma Sauva, Sophie Saffi
}

\section{To cite this version:}

Virginie Culoma Sauva, Sophie Saffi. Il possessivo e gli avverbi di luogo nel fumetto italiano: dal progetto di ricerca all'uso pedagogico. Italica Wratislaviensia , 2018, Problemi di linguistica e glottodidattica italiana, 2 (9), pp.219-237. 10.15804/IW.2018.09.24 . hal-02119236

\section{HAL Id: hal-02119236 \\ https://hal-amu.archives-ouvertes.fr/hal-02119236}

Submitted on 3 May 2019

HAL is a multi-disciplinary open access archive for the deposit and dissemination of scientific research documents, whether they are published or not. The documents may come from teaching and research institutions in France or abroad, or from public or private research centers.
L'archive ouverte pluridisciplinaire HAL, est destinée au dépôt et à la diffusion de documents scientifiques de niveau recherche, publiés ou non, émanant des établissements d'enseignement et de recherche français ou étrangers, des laboratoires publics ou privés. 
Saffi, S. \& Culoma Sauva, V. (2018). Il possessivo e gli avverbi di luogo nel fumetto italiano: dal progetto di ricerca all'uso pedagogico. Italica Wratislaviensia, 9(2), 219-237.

DOI: http://dx.doi.org/10.15804/IW.2018.09.24

Sophie Saffi \& Virginie Culoma Sauva

Università di Aix Marseille

\title{
IL POSSESSIVO E GLI AVVERBI \\ DI LUOGO NEL FUMETTO ITALIANO: \\ DAL PROGETTO DI RICERCA ALL'USO PEDAGOGICO
}

\section{THE POSSESSIVE AND THE ADVERBS OF PLACE IN THE ITALIAN COMIC BOOK: FROM THE RESEARCH PROJECT TO THE PEDAGOGICAL USE}

\begin{abstract}
Since 2013, within the CAER Research Unit, we have been producing corpus of Disney comics (Topolino) and Italian Graphic Novels. A longitudinal study $(30 \mathrm{~s}, 90 \mathrm{~s}, 21 \mathrm{st}$ Century) allows us to compare the Italian standard and the various regional achievements. The comic introduces a new class of written language that reproduces the speech and alters the classic dichotomy written vs. spoken. Dialogue in comics is enriched with oral representations rarely present in literary or print texts. We suggest the results of a diachronic, synchronous and contrastive study of the use of possessive and place adverbs related to demonstrative in the standard systems of Italian and French, validating the hypotheses considered in previous studies. The aim is to propose the physiognomy of the Italian in relation to the explicit and implicit brand of ownership and the most recurring substitution strategies, including the reflexive solution. In fact, what is distinguished is the part of activity that is opposite to passivity: the power of animation will then be at the center of our interest. We also look at the results of the use of place adverbs for demonstrative and propose the hypothesis of an evolutionary movement of the conception of person and space in contemporary Italian. Finally, we present the use of comic book as an inverse pedagogy tool in our Italian language instruction addressed to three-year graduate.
\end{abstract}

Keywords: comics, possessive, adverbs of place 
$\mathrm{D}$ 2013 costruiamo corpora di fumetti Disney e Graphic Novel itaiani. Uno studio longitudinale (dagli anni ' 30 a oggi) ci permette di confrontare l'italiano standard e le varie realizzazioni regionali. Si tratta di elaborare dei corpora orali e scritti della lingua nazionale in ogni regione, e di studiare i vari paradigmi (dimostrativi, avverbi e preposizioni di luogo, possessivi, pronomi personali, ecc.) in un intento comparativo con l'obiettivo di valutare così il livello di autonomia o meno delle varianti regionali rispetto allo standard della lingua. L'esame di corpora reali fa da legame tra rappresentazioni in lingua e manifestazioni in discorso, in un approccio teorico guillaumiano di psicomeccanica del linguaggio ${ }^{1}$. Il nostro corpus scritto registra a tutt'oggi una cinquantina di opere e una trentina di numero di riviste.

Con il presente lavoro, intendiamo esporre il nostro materiale di ricerca, i risultati ottenuti sui possessivi e sugli avverbi di luogo, nonché due esempi di sfruttamento didattico di un simile materiale.

\section{IL FUMETTO COME MATERIALE SCIENTIFICO}

I fumetti Disney condividono la stessa caratteristica: il mantenimento nel tempo di un livello di linguaggio standard che non lascia mai il posto a un linguaggio familiare anche se il registro della lingua è informale. Questo fatto consente, nell'ambito di uno studio linguistico, di separare ciò che tocca al diafasico da una parte e al genere Fumetto dall'altra. In questo modo, è possibile constatare che il fumetto introduce nuove

1 La nostra problematica generale si organizza attorno alla ricerca di una comprensione approfondita della sistematica italiana secondo i principi teorici e metodologici guillaumiani. Per Gustave Guillaume, l'atto di linguaggio è un'operazione di pensiero alla base di ogni produzione del linguaggio, la sua descrizione ci permette di capire l'intero sistema studiato e il suo funzionamento. Secondo Gustave Guillaume, l'atto di linguaggio è diviso in due sistemi separati ma interdipendenti, consequenziali, ma comunque distinti: la lingua e il discorso. Segue quindi la distinzione saussuriana langue/parole (Guillaume, 1971, p. 19; Saffi, 2010, pp. 17-24). Guillaume distingue l'atto della constatazione dall'atto della comprensione, il primo mostra la realtà superficiale delle apparenze, ampia e disorganizzata cioè "un immenso non-sistema"), il secondo porta alla comprensione della realtà profonda e consente di afferrarne la coerenza. 
categorie per lo scritto che riproduce l'orale e perturba la classica dicotomia diamesica (scritto vs parlato). Per esempio, i dialoghi del fumetto segnano le disfluenze raramente presenti nei testi letterari o di stampa: le interruzioni dovute alla sorpresa, l'imbarazzo, la menzogna ecc.

L'autore di fumetti cerca di canalizzare, anzi di fissare, l'interpretazione del lettore per mantenerla il più vicino possibile a ciò che lui ha deciso di comunicare. Il testo, nei fumetti, utilizza la punteggiatura regolare. Si nota anche una proliferazione di punti esclamativi che, nei numeri degli anni 2000, sono quasi assimilati a dei punti finali di frase, come se ci fosse la necessità di un marchio specifico dell'accento di frase legato alla prosodia.

Il genere Fumetto-Graphic Novel presenta la specificità di essere un'arte del racconto in immagini, così come l'arte cinematografica, e quindi di essere fondato sul legame tra testo e immagine. Il genere si è specializzato con una standardizzazione dei soggetti e delle forme e l'apparizione almeno in ambito francofono di una vera e propria cultura del fumetto in Francia negli anni ' 80 , quando la cultura underground è diventata cultura dominante. Il Graphic Novel è una categoria nata dalla porosità tra fumetto e letteratura e da iniziative di autori underground che risalgono agli anni ' 60 e '70 le cui produzioni presentano le caratteristiche comuni di non ricercare un bel disegno, di indirizzarsi a un lettore adulto e di trattare tematiche difficili. Il Graphic Novel italiano si rivendica come un movimento letterario con una strategia propria per diffondersi negli scaffali letterari delle librerie tramite l'adozione di un formato diverso da quello del fumetto e più vicino a quello di un romanzo.

Per la parte Disney, i nostri corpora sono anche composti da numeri di Nel regno di Topolino degli anni '30 e di Topolino degli anni ' 90 . Si tratta di un misto di creazioni originali e di traduzioni. Daniela Pietrini (2009), basandosi su un corpus di fumetti Disney pubblicati in Italia dagli anni ' 50 ad oggi, considera la varietà d'italiano costituita dal linguaggio del fumetto come una sorta di specchio delle modifiche e degli sviluppi dell'italiano informale degli ultimi cinquant'anni. Il marchio di fabbrica di Disney consiste nella conservazione di un livello di lingua standard e in contenuti non polemici e apolitici. Tale 
programma mantiene i fumetti Disney distinti dagli altri fumetti. Questa distinzione si riscontra tanto nei confronti dei Graphic Novels che mirano a un pubblico adulto quanto nei confronti dei fumetti indirizzati ai bambini. I nostri corpora vengono tratti da questo insieme di testi, che includono i testi di arrivo di traduzioni, perché sono sempre molto omogenei e riflettono la lingua standard accettata dalla comunità linguistica, il che ci consente di proporre ipotesi sulla rappresentazione spaziale nella lingua standard, ma nel genere specifico delle "scritture brevi".

\section{L'ESPRESSIONE DELL'APPARTENENZA}

Suggeriamo i risultati di uno studio diacronico, sincronico e contrastivo dell'uso del possessivo nei sistemi standard dell'italiano, che convalidano le ipotesi considerate in studi precedenti. L'obiettivo è quello di delineare la fisionomia dell'italiano rispetto al marchio esplicito e implicito della possessione e le strategie di sostituzione più ricorrenti, tra cui la soluzione riflessiva. Infatti, ciò che si distingue è la parte di attività opposta alla passività: il potere di animazione sarà quindi al centro del nostro interesse. Abbiamo scelto di concentrarci su un aspetto in particolare attraverso lo studio di un esempio specifico: l'indicazione, nel discorso dell'appartenenza, in italiano per occorrenze che rientrano abitualmente nell'ambito della sfera personale, item per i quali l'italiano di solito non usa il possessivo. Ci interessa in particolar modo di mettere alla prova l'ipotesi secondo cui in un contesto interlocutivo molto forte, si svolgeranno discussioni fra i vari membri presenti, discussioni che potrebbero permetterci di migliorare quello che già sappiamo sulle rappresentazioni della persona nella lingua considerata. 
Figura 1: Peppino Impastato (Rizzo \& Bonaccorso, 2010, p. 31)

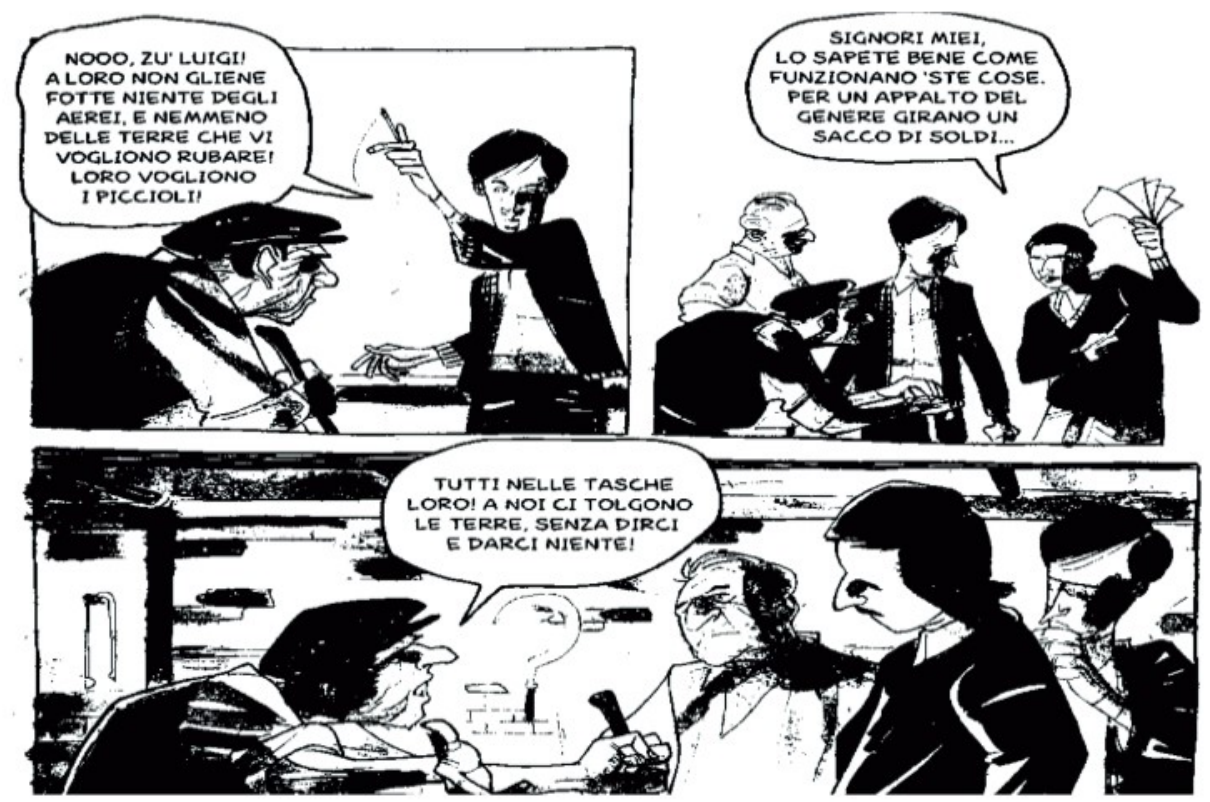

Prima di esaminare l'espressione esplicita dell'appartenenza in italiano nell'ambito della sfera personale, conviene tornare velocemente sulla definizione di questo concetto. Charles Bally è stato uno dei primi a studiare la problematica della possessione alienabile/inalienabile, secondo i termini usati da Lucien Lévy-Bruhl (1916), che definisce così questa nozione:

La sfera personale comprende o può comprendere cose e esseri associati a una persona in un modo abituale, intimo, organico (per es. il corpo e le sue parti, i vestiti, la famiglia, ecc.). Ogni elemento costitutivo della sfera personale è considerato, non come una semplice proprietà, ma come una parte integrante della persona. (Bally, 1926, p. 68)

Abbiamo rilevato sei categorie per le quali l'italiano all'interno di un discorso può fare a meno dell'uso del possessivo per esprimere l'appartenenza: i legami di parentela, la parentela estesa, le parti del corpo, gli oggetti personali concreti, gli oggetti personali virtuali e gli attributi 
della persona. Tuttavia, abbiamo anche riscontrato alternanze tra occorrenze nelle quali compare il possessivo per la parentela vicina e occorrenze nelle quali non viene espressa in modo esplicito, ma solo tramite l'articolo determinativo ad esempio. Quali sono allora i meccanismi in gioco quando il possessivo compare? Abbiamo scelto, per una ragione di tempo, di incentrarci sullo studio di un esempio rilevante.

FIGURA 2: Spartizione della possessione implicita in italiano

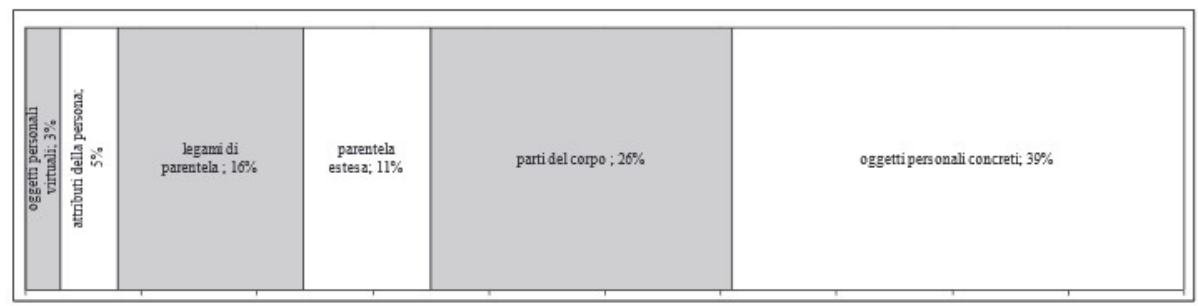

Nel Graphic Novel intitolato Rina Fort, troviamo questo tipo di alternanza: due elementi di contesto: Rina, una friulana di 31 anni, è passata alla storia come "la belva di via San Gregorio". Il 30 novembre 1946 uccide la famiglia dell'amante aggredendola a colpi di spranga. Negherà poi di aver ucciso quei bambini anche in punto di morte. Il possessivo ha in questi casi secondo noi, una funzione di enfasi legata alla spazialità.

FIGURA 3: Rina Fort (Rizzotto \& Vivaldo, 2006, p. 27)
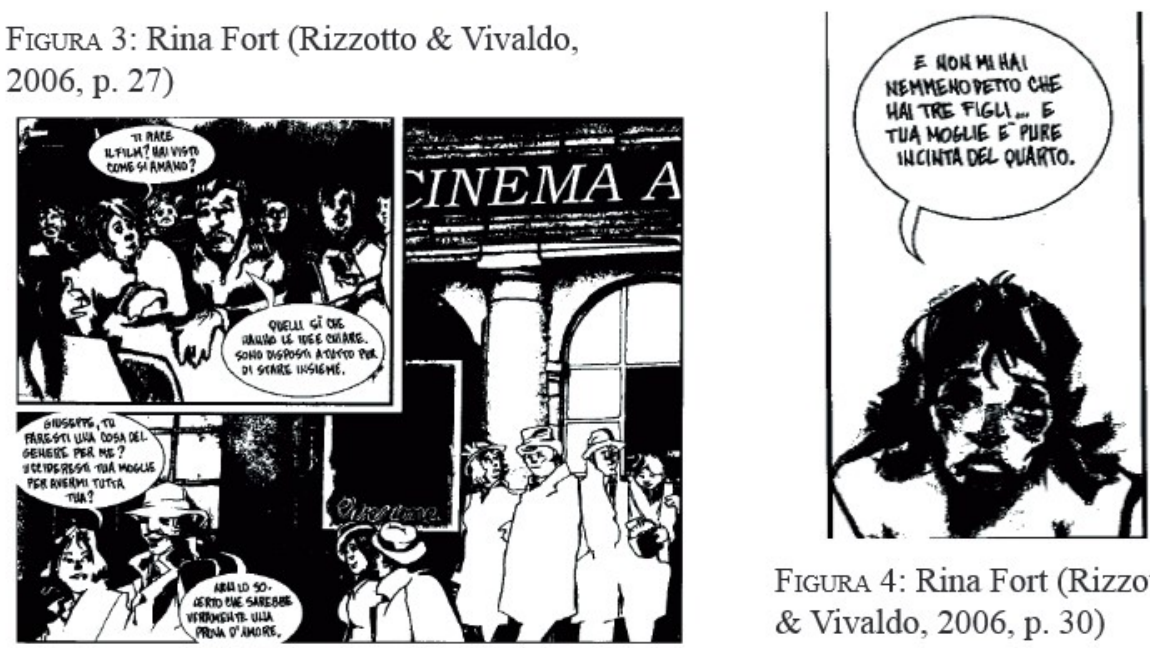

FIGURA 4: Rina Fort (Rizzotto \& Vivaldo, 2006, p. 30) 
In questi esempi il possessivo permette al locutore di segnare la sua posizione rispetto a quello che dice. Infatti, è evidente, nel contesto di una discussione tra un uomo sposato e l'amante, che i possessivi hanno un forte valore espressivo. Qui abbiamo l'impressione che sono discusse le appartenenze rispettive di ogni interlocutore al momento stesso della conversazione.

L'aggettivo possessivo Tua accusatorio, nelle occorrenze RF11 e RF14, permette alla parlante (Rina) di evidenziare il fatto che la moglie dell'amante non appartiene allo spazio che stanno condividendo, ma solo a quello di quest'ultimo.

FiguRA 5: Rina Fort (Rizzotto \& Vivaldo, 2006, p. 30) RF 15: "Io non amo mia moglie" (parla l'amante Pippo)

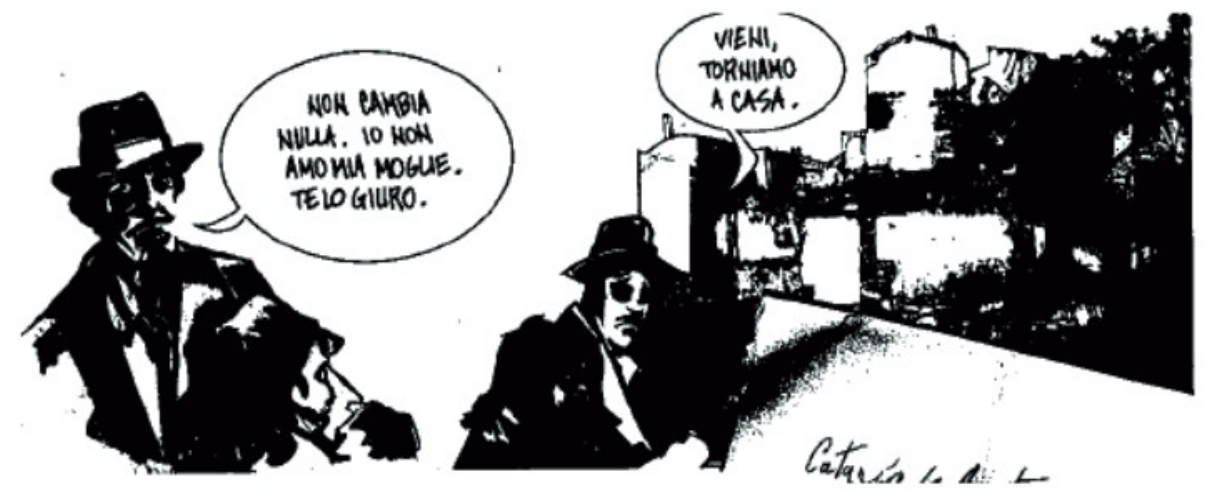

Mia, item 15 è usato mentre la persona del possessore compare due volte tramite il pronome personale soggetto $I o$ e la persona del predicato amo, ma il possessivo che segue non ha per niente un valore affettivo, che sarebbe alquanto sgradito nell'ambito di questa conversazione. Il locutore cerca di convincere l'amante a non lasciarlo. Il possessivo è allo stesso tempo un modo per accettare le ridefinizioni spaziali proposte da Rina, ma anche un modo per lui di manifestare il divario che esiste tra i sentimenti attesi nei confronti dell'oggetto posseduto - qui sua moglie - e quelli che prova in realtà. 
La rappresentazione della persona in lingua ha quindi delle conseguenze importanti sul sistema della lingua considerata e anche sugli effetti di senso nel discorso.

FIGURA 6: Rina Fort (Rizzotto, Vivaldo, 2006, p. 33) RF 22: "Mi fa schifo la signora Franca e tutti i suoi bambini!" (parla Rina)

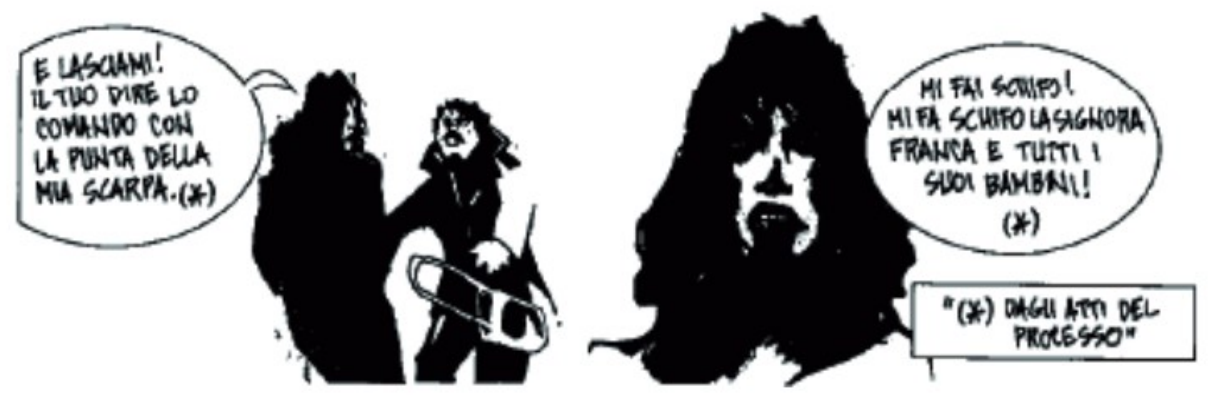

L'elemento che emerge chiaramente dall'analisi del corpus è che l'articolo determinativo viene usato quando lo spazio interlocutivo è condiviso consensualmente. Quando viene reintrodotto il possessivo in italiano, si rinegoziano e si ridefiniscono allo stesso tempo gli spazi devoluti ad ogni membro presente. L'espressione esplicita del possessivo non permette solo l'identificazione della possessione. Ogni sistema di lingua esprime così numerose sfumature il cui senso non si offre sempre subito davanti agli occhi di quello che lo studia.

\section{GLI AVVERBI DI LUOGO}

Esaminiamo anche i risultati relativi all'uso degli avverbi di luogo afferenti ai dimostrativi e proponiamo l'ipotesi di un movimento evolutivo della concezione della persona e dello spazio in italiano contemporaneo. Abbiamo già detto perché il corpus ci permette di eliminare ciò che dipende dal livello di lingua formale $v$ s informale nella marcatura spaziale, per conservare solo quel che dipende dal genere fumetto, il che ci permette di proporre ipotesi sulla rappresentazione spaziale in lingua standard ma nel genere fumetto. Abbiamo esaminato gli avverbi di luogo e le loro combinazioni con i dimostrativi. Abbiamo osservato una stabilità della frequenza degli avverbi dagli anni '30 agli anni 2000. 
FIGURA 7: Avverbi afferenti ai dimostrativi dagli anni ‘30 agli anni 2000

\begin{tabular}{l|c|c}
\hline \multicolumn{2}{c}{ FR } \\
\hline JDM & $1934-1937$ & $0,4 \%$ \\
\hline JDM & $2011-2012$ & $0,4 \%$ \\
\hline Rwanda 1994 & 2005 & $0,6 \%$ \\
\hline \multicolumn{3}{|c}{ IT } \\
\hline TOP & $1936-1939$ & $0,5 \%$ \\
\hline TOP & $1995-1998$ & $0,4 \%$ \\
\hline Peppino Impastato & 2010 & $0,6 \%$ \\
\hline Porto Marghera & 2007 & $0,1 \%$ \\
\hline
\end{tabular}

Il confronto francese-italiano evidenzia delle evoluzioni differenziate: la forma più usata in italiano standard si riferisce a uno spazio prossimo puntuale, mentre in francese la forma più usata si riferisce a uno spazio esteso. La predominanza dell'avverbio puntuale in italiano è complementare di un sistema binario di dimostrativi (questo vs quello) che oppone due spazi: un primo spazio, luogo dell'interlocuzione, e un secondo spazio fuori interlocuzione. I due spazi sono chiaramente delimitati, l'interlocuzione è associata a uno spazio di prossimità la cui frontiera si concretizza con l'interlocutore. Al di là di questo limite si dispiega lo spazio lontano fuori interlocuzione.

FIGURA 8: Avverbi afferenti ai dimostrativi in IT e FR

\begin{tabular}{l|c|c|c|c}
\hline IT & $q u i$ & $q u a$ & $l \grave{l}$ & $l \grave{a}$ \\
\hline Peppino Imp. 2010 & $26 \%$ & $26 \%$ & $13 \%$ & $34 \%$ \\
\hline TOP 1998 & $72 \%$ & - & $14 \%$ & $14 \%$ \\
\hline Porto Margh. 2007 & $86 \%$ & - & - & $14 \%$ \\
\hline FR & $i c i$ & $l \grave{a}$ & \multicolumn{2}{|c}{ là-bas } \\
\hline Rwanda 2005 & $28 \%$ & $72 \%$ & \multicolumn{2}{|c}{-} \\
\hline JDM 2011-12 & $29 \%$ & $70 \%$ & \multicolumn{2}{|c}{$1 \%$} \\
\hline
\end{tabular}


La sfera del locutore riguarda, secondo Marchello-Nizia, "l'appartenenza sociale, familiare o politica del locutore, una possessione propria riconosciuta, rivendicata o affermata oppure una relazione di dilezione affermata" (2004, p. 81; trad. nostra). All'interno della sfera interlocutiva della coppia dialogica che il locutore sintetizza nella propria persona, si suppone che ogni oggetto e persona siano in rapporto intimo con il locutore. Questa sfera di vicinanza e di intimità è lo spazio privilegiato dei rapporti simbiotici.

Riteniamo che la sfera estesa della persona in italiano abbia come conseguenza semiologica un abbondante ricorso allo strumento di puntamento qui ogni volta che è necessario controbilanciare la tendenza inclusiva per ottenere la rappresentazione di rapporti esterni nel discorso. Al contrario, in francese, la sfera della persona è ridotta ai soli limiti del corpo (il che implica un uso abbondante di possessivi), c'è un solo dimostrativo generico $c e$, le varietà di spazio (prossimo vs lontano) sono state ridotte a beneficio di un unico spazio generalizzato in seno al quale si sviluppano rapporti esterni. Riteniamo che la sfera ridotta della persona in francese abbia come conseguenza semiologica un abbondante ricorso al deittico esteso là ogni volta che è necessario controbilanciare la tendenza esclusiva, delimitare uno spazio condiviso e rinforzare la sutura interlocutiva.

L'indicazione spaziale perdura, ma è incentrata sulla distinzione tra inclusione / esclusione dalla sfera del locutore. La distribuzione delle forme avverbiali è illuminante: qui, la forma più usata in italiano standard, si riferisce a uno spazio prossimo puntuale. In francese la forma più usata invece è là, che si riferisce a uno spazio esteso senza riferimento alla distanza. Proponiamo l'ipotesi secondo la quale la predominanza degli usi di qui in italiano è legata alla sfera estesa della persona che favorisce rappresentazioni spaziali inclusive e dunque il ricorso alla forma puntuale permette di ottenere la rappresentazione di rapporti esterni nel discorso. Al contrario, la sfera ridotta della persona in francese favorisce rappresentazioni spaziali esclusive e quindi il ricorso alla forma estesa permette di ottenere la rappresentazione di uno spazio condiviso e di rapporti inclusivi nel discorso. 


\section{IL FUMETTO MATERIALE PEDAGOGICO}

Presentiamo adesso l'uso del fumetto in un percorso d'iniziazione alla linguistica italiana indirizzato a studenti di laurea triennale del secondo anno (18 ore divise in 12 sedute per ogni semestre). Questa lezione è anche aperta agli studenti non specialisti che hanno studiato l'italiano al liceo: questi studenti opzionali rappresentano l' $80 \%$ della decina di iscritti. Alla fine del semestre gli studenti restituiscono un power-point con la trascrizione del fumetto, una breve presentazione dell'autore e dell'opera, il corpus, i risultati e l'analisi (Fig. 9-13). Si propone una classe capovolta e in classe si comincia subito con la pratica: ogni studente trascrive un Graphic Novel italiano che sceglie in una lista e lo si aiuta ad elaborare un corpus di dimostrativi e avverbi di luogo afferenti a partire dalla sua trascrizione. Parallelamente, metà del tempo è dedicato a presentazioni di temi importanti per la comprensione delle tematiche e riflessioni in linguistica. Dopo 5 sedute, vengono presentati agli studenti il lavoro e le ipotesi dell'équipe di ricercatori. Gli studenti hanno dovuto affrontare vari dubbi durante l'elaborazione del corpus e sono tutti molto più ricettivi alle spiegazioni che nel contesto classico delle lezioni in cui si comincia con la presentazione teorica seguita poi da una messa in pratica. La seconda parte del semestre è dedicata al calcolo e analisi dei risultati dell'insieme dei corpus degli studenti e al paragone con i risultati dei ricercatori. Un confronto con la traduzione francese delle occorrenze permette di arricchire l'analisi, mentre parallelamente, nella seconda parte delle lezioni, si proseguono le presentazioni delle teorie di linguistica moderna. Le competenze in italiano degli studenti sono di livello B2, ma si constata che riescono ad avere una vera e propria riflessione sul testo: anzi l'analisi grammaticale permette loro di progredire tanto in italiano quanto in francese. 
FIgURA 9: Esempio di lavoro di studente (1)

\begin{tabular}{|c|c|l|}
\hline \multicolumn{2}{|c|}{ Tabella dei dimostrativi } \\
\hline Numero item & Pagina & \multicolumn{1}{|c|}{ Esempio } \\
\hline Reb 1 & 5 & questo è l'ultimo pezzo \\
\hline Reb 2 & 10 & te lo dico chi è quello che ti corre dietro nelle catacombe \\
\hline Reb 3 & 12 & E queste sono le chiavi \\
\hline Reb 4 & 13 & stanno organizzando una festa per il compleanno di toni, questo sabato \\
\hline Reb 5 & 23 & in questo modello, l'atomo era immaginato come elettricamente neutro \\
\hline Reb 6a & 24 & $\begin{array}{l}\text { e questo nucleo è circondato da cariche negative che gli vorticano } \\
\text { intorno }\end{array}$ \\
\hline Reb 6b & 24 & $\begin{array}{l}\text { con Bhor si comprenderà come queste cariche negative non siano disor- } \\
\text { dinate nella nube intorno al nucleo }\end{array}$ \\
\hline Reb 7a & 26 & questa è una foto di classe di quando studiavo a Gottinga \\
\hline Reb 7b & 26 & eccomi qua \\
\hline Reb 8a & 27 & $\begin{array}{l}\text { ma lo sai che le minigonne di quegli anni erano molto più corte di quelle } \\
\text { che si usano adesso ?! }\end{array}$ \\
\hline
\end{tabular}

FIGURA 10: Esempio di lavoro di studente (2)

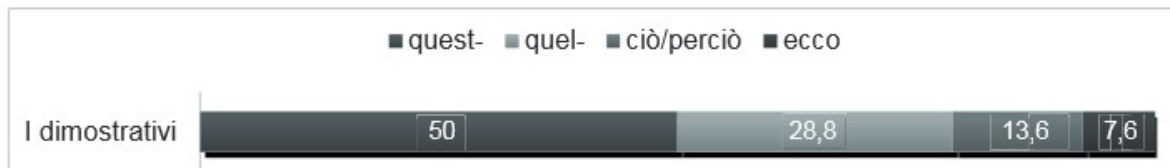

FIGURA 11: Esempio di lavoro di studente (3)

\begin{tabular}{|c|c|c|}
\hline \multicolumn{2}{|c|}{ Tabella degli avverbi di luogo } \\
\hline Numero item & Pagina & Esempio \\
\hline Reb 63a & 26 & e qui ? \\
\hline Reb 63b & 26 & eccomi qua \\
\hline Reb 64a & 27 & e qui ? \\
\hline
\end{tabular}




\begin{tabular}{|c|c|c|}
\hline \multicolumn{2}{|c|}{ Tabella degli avverbi di luogo } \\
\hline Numero item & Pagina & Esempio \\
\hline Reb 64b & 27 & qui ero con la nonna \\
\hline Reb 65 & 38 & eccole qui \\
\hline Reb 66 & 100 & Che bello stare qua $!$ \\
\hline Reb 67 & 119 & Siamo arrivati qui parlando di archetipi \\
\hline Reb 68 & 120 & Ed ecco li \\
\hline Reb 69a & 122 & Gli amici sconisciuti sono li per questo \\
\hline Reb 69b & 122 & Che bello stare qua \\
\hline
\end{tabular}

FIGURA 12: Esempio di lavoro di studente (4)

\begin{tabular}{|c|c|c|c|c|}
\hline \multicolumn{5}{|c|}{ =qui $=$ qua $=$ lì "là } \\
\hline Gli avverbi di luogo & 50 & 30 & 20 & 0 \\
\hline
\end{tabular}

FIGURA 13: Esempio di lavoro di studente (5)

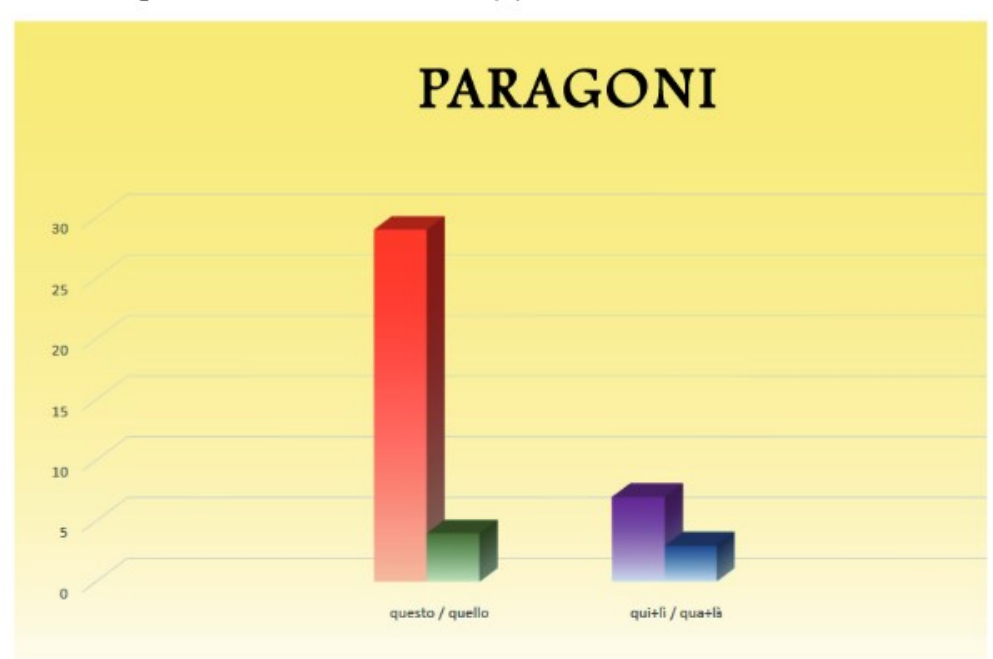


Per fare un esempio, in questa vignetta il pronome $c h i$ è stato analizzato dalla studentessa prima in quanto dimostrativo e, successivamente, in quanto relativo. Il che si spiega col fatto che, secondo Dardano e Trifone (2010, p. 261) "Chi è un pronome doppio, in quanto unisce in sé la funzione di due pronomi diversi: uno dimostrativo [...] o indefinito [...], l'altro relativo [...]. Per questa sua particolarità è l'unico fra i pronomi relativi che si può usare in forma assoluta". La traduzione letterale in francese comincia con ceux qui...: l'analisi grammaticale di queste parole ha richiesto un momento di riflessione alla studentessa (e un po' di aiuto), ma il confronto con l'italiano ha provocato la curiosità e lo sforzo di comprensione. Quindi per noi l'obbiettivo era stato raggiunto.

FIGURA 14: Sereno su gran parte del paese (Scoppetta, 2009, p. 32)

SER59: - "Ehilà, amico! Chi non muore si rivede!"2

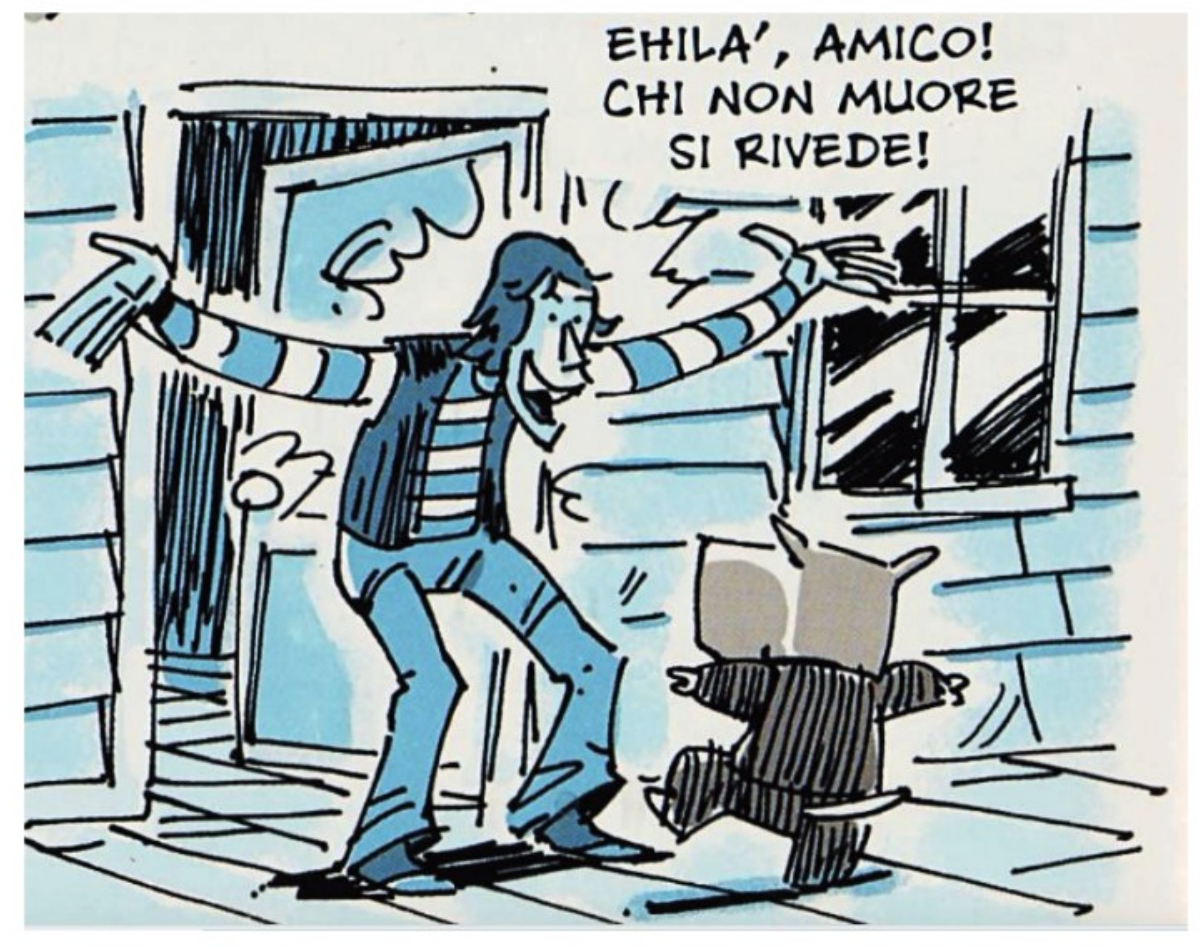

2 Trad. francese: Hé toi, mon ami! On se reverra! Trad. letterale: Ceux qui ne meurent pas sont destinés à se revoir. 
Inoltre nell'ambito del percorso di laurea triennale ${ }^{3}$, abbiamo proposto un workshop con universitari e liceali dedicato al Graphic Novel. Si trattava di presentare il genere Graphic Novel, la specificità della lingua usata con la combinazione dell'immagine e dello scritto, le varie forme di espressione dello scritto con le nuvolette o balloon, le didascalie, ma anche i pertesti, cioè i testi contenuti nelle immagini all'infuori delle nuvolette o delle didascalie ${ }^{4}$.

Dopodiché una professionista, direttrice di una start up di edizione numerica, è intervenuta ed ha presentato il suo lavoro per quanto riguarda il media considerato. Il che ha aiutato gli alunni (universitari e liceali) a fare un legame chiaro tra le competenze in via di acquisizione e quelle che dovranno avere sul mercato del lavoro. È stato interessante per loro capire come potrebbero essere valorizzate le loro capacità di esperti di una lingua e di una cultura straniera.

La terza parte di questo seminario è stata dedicata allo sfruttamento didattico di alcune pagine di una Graphic Novel sulla Grande Guerra intitolata La Grande Guerra, Storia di nessuno (Di Virgilio \& Pascutti, 2008). A fine giornata è stato dato agli alunni un compito di espressione scritta la cui direttiva era Scrivere la lettera della moglie che risponde a suo marito sul fronte, in 150 parole. Dovevano usare il lessico dell'esercito, del fronte, i codici della lettera a un membro della propria famiglia sul modello evidenziato sottostante, studiato in classe, di una lettera di soldato mandato a sua moglie. I loro lavori saranno pubblicati sul sito del liceo.

3 Aix Marseille Université propone una laurea triennale in lingue e letterature straniere con indirizzo specialistico in studi italiani (Licence LLCER - Études italiennes).

4 Per una definizione teorica si veda Manco, 2018. 
FIGURA 16: La Grande Guerra (Di Virgilio, Pascutti, 2008, pp. 11-12)
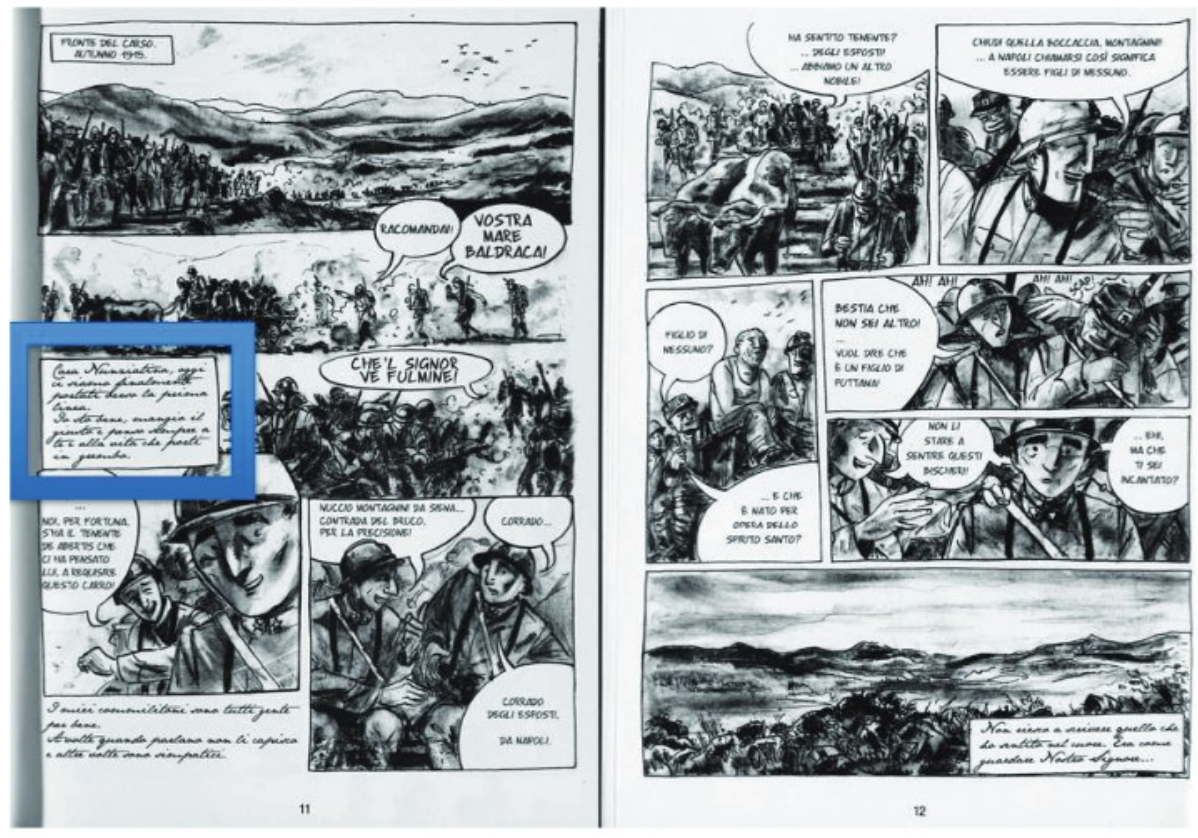

Al terzo anno, il percorso prosegue proponendo agli studenti un lavoro simile ma che integra uno studio dei possessivi o altri elementi della rappresentazione spaziale, e una riflessione sulla rappresentazione scritta dell'orale. Nella loro conclusione devono inoltre valutare i benefici del percorso, la maniera in cui hanno superato gli ostacoli, e anche i possibili miglioramenti. Secondo gli studenti, la pratica consente loro: di contestualizzare i principi teorici della lezione, di rendersi conto che una ipotesi può essere verificata ma anche discussa, di rinforzare le loro conoscenze della grammatica francese e di migliorare la loro espressione in francese grazie all'approccio comparativo con l'italiano, di migliorare le loro competenze in informatica grazie all'elaborazione dei diagrammi e delle tabelle, e del manipolare diverse opzioni di word, di acquisire il rigore e la pazienza richieste dalla verifica della trascrizione e del corpus (ogni studente deve verificare il lavoro di un suo collega).

Gli studenti apprezzano particolarmente: il lavoro sui fumetti, la traduzione dei fumetti che li conduce a una comprensione sottile della 
costruzione sintattica nelle due lingue, a non focalizzare sui morfemi ma a prendere in considerazione l'enunciato globale e, per esempio, a scoprire certe espressioni idiomatiche; apprezzano il lavoro collaborativo, soprattutto quando hanno avuto l'occasione di fare binomio con uno studente Erasmus.

Per concludere, speriamo di avere dimostrato che il materiale particolare costituito dal fumetto favorisce la realizzazione di nessi tra la ricerca e la didattica. Nell'ambito della ricerca, questo strumento si è rivelato molto utile per uno studio linguistico incentrato sulla spazialità grazie all'iconografia e alla specificità della lingua e ci ha permesso di farci un'idea più precisa ancora dell'impatto della rappresentazione della persona sul discorso. Nell'area didattica, il fumetto, grazie all'iconotestualità, è risultato molto stimolante per gli studenti che, messi in condizione di creare loro stessi un corpus, hanno potuto riflettere sulla restituzione di un contenuto da una lingua all'altra e hanno potuto contestualizzare al più presto la teoria, si sono mostrati motivati e capaci di una comprensione sottile di principi teorici complessi.

\section{BIBLIOGRAFIA}

Bally, C. (1926). L'expression des idées de sphère personnelle et de solidarité dans les langues indo-européennes. In F. Funkhauser \& J. Jud (eds.), Festschrift Louis Gauchat (pp. 68-78). Aarau: H.R. Sauerlander.

Culoma Sauva, V. (2014). Étude diachronique et psychosystématique des possessifs et de la représentation spatiale en italien, français et roumain. $\mathrm{PhD}$ thesis. CAER EA 854, ED 355, Aix Marseille Univ.

Culoma Sauva, V. (2015). Les possessifs et la représentation de l'espace du locuteur en italien et dans les langues romanes. Studii de Ştiinţă şi Cultură, XI, n³, 49-56.

Di Virgilio, A. \& Pascutti, D. (2015). La Grande Guerra, Storia di nessuno. Padova: Edizioni BeccoGiallo.

Dardano, M. \& Trifone, P. (2010). La nuova grammatica della lingua italiana $\left(1^{a}\right.$ ed. 1997). Bologna: Zanichelli.

Gadet, F. (2004). La signification sociale de la variation. Romanistisches Jahrbuch Band, 54, 98-114. 
Lage, M.J., Platt, G. \& Treglia, M. (2000), Inverting the Classroom, A Gateway to Creating an Inclusive Learning Environment. The Journal of Economic Education, vol. 31, $\mathrm{n}^{\circ} 1,30-43$.

Manco, A. (2018, to appear). La pertestualità: principi teorici, applicazioni, traduzione. Cahiers d'Études Romanes, 12.

Pietrini, D. (2009). Parola di Papero. Storia e tecniche della lingua dei fumetti di Disney. Firenze: Franco Cesati.

Rizzo, M., Bonaccorso, L. (2010). Peppino Impastato. Un giullare contro la mafia. Padova: BeccoGiallo.

Rizzotto, M. \& Vivaldo, A. (2006). Rina Fort, la donna che passò alla storia come la belva di San Gregorio. Padova: BeccoGiallo.

Saffi, S. (2010). La personne et son espace en italien. Limoges: Lambert-Lucas.

Saffi, S. (2012). Fumetti e rappresentazione semiologica dello spazio. In A. Manco (ed.), Comunicazione e Ambiente (Parte seconda: Comunicazione e graphic novel; pp. 221-34). Napoli: Università degli Studi di Napoli "L'Orientale".

Saffi, S. (2014). De l'intérêt d'une étude contrastive des bandes dessinées Topolino et Le journal de Mickey. Studia Universitatis Babeș-Bolyai Philologia, LIX, $\mathrm{n}^{\circ} 3,7-23$.

Saffi, S. (2014). La concezione spaziale a prova di traduzione italiano-francese, francese-italiano: illustrazioni morfo-sintattiche e lessicali nelle traduzioni di una graphic novel e di un saggio di linguistica teorica. Kwartalnik neofilologiczny, $\mathrm{nr}$ 1, 125-39.

Saffi, S. (2015). La représentation spatiale en italien et en français : étude contraste des démonstratifs et adverbes de lieu afférents. Studii de Ştiinţă şi Cultură, XI, n³, 57-66.

Saffi, S. \& Culoma Sauva, V. (2015). L'image du soldat de la grande guerre dans des bandes dessinées italiennes et françaises des années 90 à aujourd'hui. Italies, L'image du soldat au XXe siècle, $\mathrm{n}^{\circ} 19,133-55$.

Sams, A. \& Bergmann, J. (2014). La classe inversée. Technologie de l'éducation. Repentigny Québec: Édition Reynald Goulet.

Scoppetta, A. (2009). Sereno su gran parte del paese, una favola per Rino Gaetano. Padova: BeccoGiallo. 
Riassunto: Dal 2013, nel quadro dell'unità di ricerca CAER, elaboriamo corpora di fumetti Disney (Topolino) e Graphic Novel italiani. Uno studio longitudinale (anni ‘ 30, ' $90, \mathrm{XXI}^{\circ}$ sec.) ci permette di confrontare l'italiano standard e le varie realizzazioni regionali. Il fumetto introduce una nuova categoria di lingua scritta che riproduce il parlato ed altera la classica dicotomia scritto vs. parlato. I dialoghi nei fumetti sono arricchiti di rappresentazioni delle disfluenze orali raramente presenti in testi letterari o di stampa. Suggeriamo i risultati di uno studio diacronico, sincronico e contrastivo dell'uso del possessivo e degli avverbi di luogo afferenti ai dimostrativi nei sistemi standard dell'italiano e del francese, che convalidano le ipotesi considerate in studi precedenti. L'obiettivo è quello di proporre la fisionomia dell'italiano rispetto al marchio esplicito e implicito della possessione e le strategie di sostituzione più ricorrenti, tra cui la soluzione riflessiva. Infatti, ciò che si distingue è la parte di attività opposta alla passività: il potere di animazione sarà quindi al centro del nostro interesse. Esaminiamo anche i risultati relativi all'uso degli avverbi di luogo afferenti ai dimostrativi e proponiamo l'ipotesi di un movimento evolutivo della concezione della persona e dello spazio in italiano contemporaneo. Infine presentiamo l'uso del fumetto come strumento in classe capovolta nel nostro insegnamento di linguistica italiana indirizzato a studenti di laurea triennale.

Parole chiave: fumetto, possessivi, avverbi di luogo 